\title{
Surgical Management of 4 Cases of Craniofacial Fibrous Dysplasia
}

\author{
Youssouf Sogoba ${ }^{*}$, Moussa Diallo1, Boubacar Sogoba1, Djènè Kourouma1, \\ Izoudine Blaise Koumaré1, Mamadou Diallo1, Oumar Coulibaly², Mahamadou Dama2, \\ Mamadou Salia Diarra3 ${ }^{3}$, Seybou Hassane Diallo4, Salimata Diallo4, Oumar Diallo3, \\ Youssoufa Maiga4, Drissa Kanikomo' ${ }^{1}$
}

\author{
${ }^{1}$ Depatment of Neurosurgery, Hôpital Gabriel Touré, Bamako, Mali \\ ${ }^{2}$ Department of Neurosurgery, Hopital du Mali, Bamako, Mali \\ ${ }^{3}$ Department of Neurosurgery, Hopital Mère-enfant, Bamako, Mali \\ ${ }^{4}$ Depatment of Neurology, Hôpital Gabriel Touré, Bamako, Mali \\ Email: ^sogobayoussouf@yahoo.fr
}

How to cite this paper: Sogoba, Y., Diallo, M., Sogoba, B., Kourouma, D., Koumaré, I.B., Diallo, M., Coulibaly, O., Dama, M., Diarra, M.S., Diallo, S.H., Diallo, S., Diallo, O., Maiga, Y. and Kanikomo, D. (2021) Surgical Management of 4 Cases of Craniofacial Fibrous Dysplasia. World Journal of Neuroscience, 11, 279-286.

https://doi.org/10.4236/wins.2021.114020

Received: August 1, 2021

Accepted: October 23, 2021

Published: October 26, 2021

Copyright $\odot 2021$ by author(s) and Scientific Research Publishing Inc. This work is licensed under the Creative Commons Attribution International License (CC BY 4.0).

http://creativecommons.org/licenses/by/4.0/

\begin{abstract}
Background: Fibrous dysplasia is an uncommon skeletal disorder in which normal bone and marrow are replaced with fibro-osseous tissue. The disease comprises $2.5 \%$ of all bone tumors and $7.5 \%$ of all benign bone neoplasm. It is the progressive, slowly developing disease and the optimum treatment remains unclear in many cases. Aim: In this study, the authors report their experience in the surgical treatment of four cases of craniofacial fibrous dysplasia. Cases presentation: The study involved 4 patients with craniofacial fibrous dysplasia. There were 3 men and a woman. The patients were 10, 17, 20 and 8 years old. No patient had a focal neurological deficit. The CT scan appearance was compatible with Fibrous dysplasia in all patients. The site of disease was frontal in one case and parietal in the other 3 cases. Cosmetic surgical treatment was performed in all patients. Cranioplasty was performed in one patient and planned for the other three. Conclusion: Fibrous Dysplasia is a benign slow growing disease that may cause as well as clinical symptom and aesthetical discomfort. Radical resection, if possible, is the only technique to obtain resolution of the disease.
\end{abstract}

\section{Keywords}

Fibrous Dysplasia, Surgical Treatment, Bone Tumor

\section{Introduction}

Fibrous dysplasia (FD) is an uncommon skeletal disorder in which normal bone 
and marrow are replaced with fibro-osseous tissue [1]. FD was first described in 1938 by Lichtenstein [2]. The disease is classified into three variants: monostotic, polyostotic, and McCune-Albrigtht syndrome [3]. The term craniofacial dysplasia (CFD) has been introduced to describe forms arising in the contiguous bones of the cranium and facial skeleton [4]. FD comprises $2.5 \%$ of all bone tumors and $7.5 \%$ of all benign bone neoplasm [5]. The monostotic form occurs most frequently and represents $75 \%$ of cases [3]. The diagnosis of FD is usually based on clinical, radiographic, and histopathological features. On computed tomography (CT) scan and radiography, the healthy bone is replaced by bone with a "ground-glass" appearance, with no visible trabecular pattern [5]. The disease is more common in children and young people and the most common presentation is swelling; other manifestations include localized pain, deformity, fractures, and compromised vision or hearing [3] [6] [7]. FD is the progressive, slowly developing disease and the optimum treatment remains unclear in many cases. Clinical observation is recommended in asymptomatic patients and surgery remains the mainstay of treatment if the disease affects normal life [8]. In this paper, the authors report their experience in the surgical management of four cases of CFD.

\section{Case 1}

A 10 year-old boy with 12 months history of swelling of the right side of the head and orbital ring was admitted to our neurosurgical department. The patient had previously consulted in ophthalmology for right fronto-orbital tumefaction. The ophthalmologic examination was without particularity with normal visual acuity. He was referred to us for his head deformity. On admission, clinical examination revealed that the patient had a Glasgow Coma Scale (GCS) at 15 with, no focal neurologic deficit. As showed in Figure 1, the patient had swelling in the right parietal and fronto-orbital bones. The CT scan (Figure 2) appearance was compatible with Fibrous Dysplasia. The routine laboratory test was normal. A radical resection of the parietal lesion and a shawing of the fronto-orbital one were performed (Figure 3). The histopathological examination confirmed FD. The postoperative course was uneventful. The cranioplasty was planned after puberty.

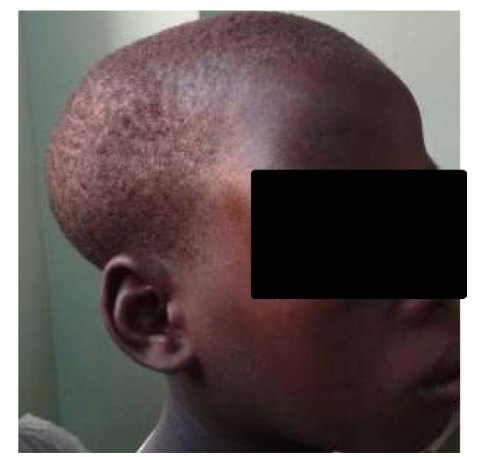

Figure 1. Clinical photograph showing the swelling in the right parietal bone. 


\section{Case 2}

This 17 year-old boy was admitted to our neurosurgical with complaining of right fronto-orbital swelling and headache. On admission, he was alert with GCS at15. Physical examination was normal except that deformity in the right side of fronto-orbital region (Figure 4). He had no visual or hearing loss. The CT scan (Figure 5) showed frontal bone anomaly which were compatible with fibrous dysplasia. Preoperative blood check was normal. Radical resection was done (Figure 6). The histopathological examination confirmed the diagnosis of FD. The patient discharged without any complication and was scheduled for cosmetic cranioplasty in the next 2 years.

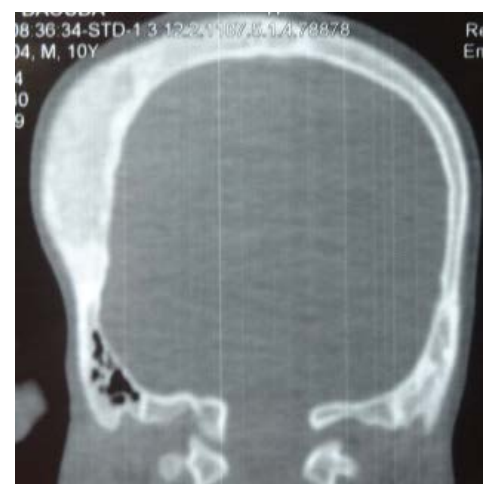

Figure 2. CT scan showing the right parietal bone with FD appearance.

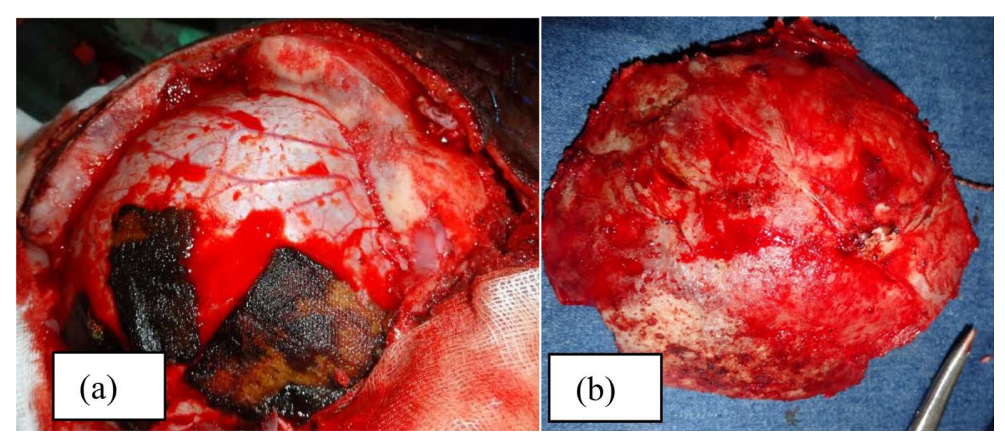

Figure 3. Operative view (a) resection of Fibrous dysplasia, (b) bone fap.

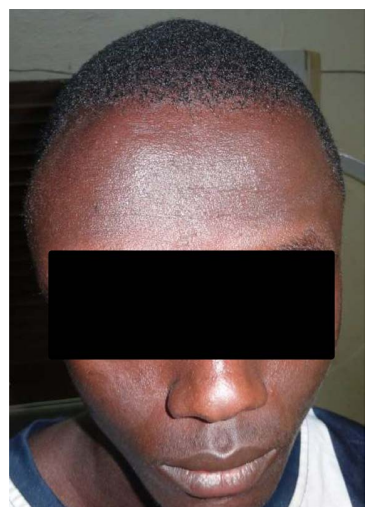

Figure 4. Clinical photograph showing the deformity in the right side of fronto-orbital region. 


\section{Case 3}

A 20 year-old male with right fronto-parietal slow growing tumefaction and headache for 2-years duration was admitted in our neurosurgical department. Physical examination was normal except the right painless frontoparietal tumefaction. The CT scan (Figure 7) showed the fibrous dysplasia appearance of the tumefaction. Radical resection of the lesion was done. The histopathological examination confirmed the diagnosis of FD. The postoperative X-ray (Figure 8) showed the bone defect. The cosmetic cranioplasty with acrylic cement was performed one year later as seen in the control X-ray (Figure 9). The post-operative course was favorable without any aesthetical discomfort.

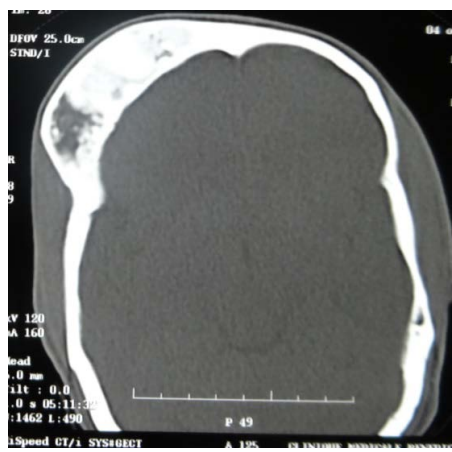

Figure 5. CT scan with FD appearance of the right frontal bone.

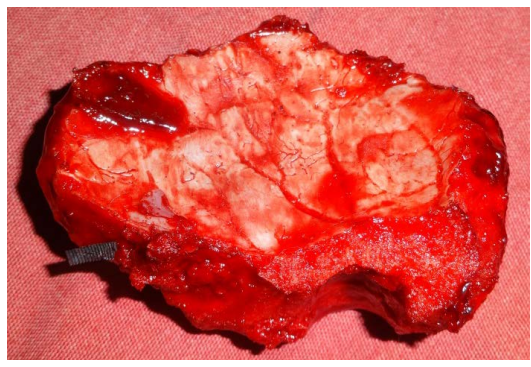

Figure 6. Bone flap after resection of FD.

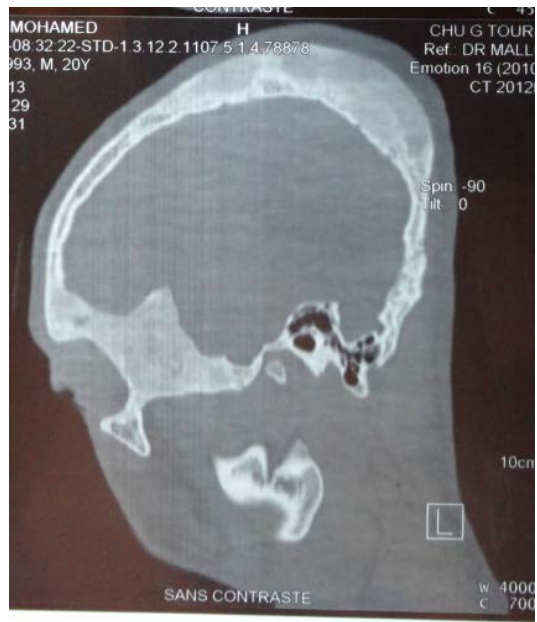

Figure 7. CT scan with FD appearance of the right parietal bone. 


\section{Case 4}

This 8 year-old girl was admitted to our neurosurgical department with 4 years history of swelling in the left parietal region of the head. Physical and neurological examinations were normal except the painless tumefaction of the left parietal bone. The clinical aspects (Figure 10) as well as the CT scan (Figure 11) features were compatible with Fibrous dysplasia. We recommended observation but her family did not agree and decided radical resection (Figure 12) due to aesthetical discomfort. The histopathological examination confirmed the diagnosis of FD. The post-operative course was uneventful. The patient was scheduled for cosmetic cranioplasty after puberty.

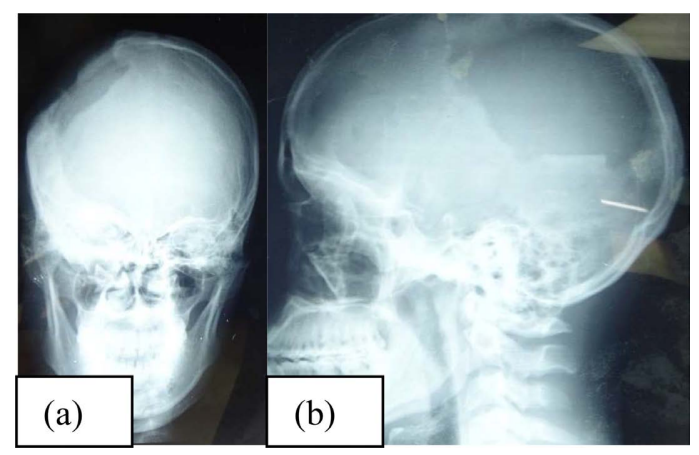

Figure 8. Post craniotomy (a) AP, (b) lateral X-ray showing the bone defect.

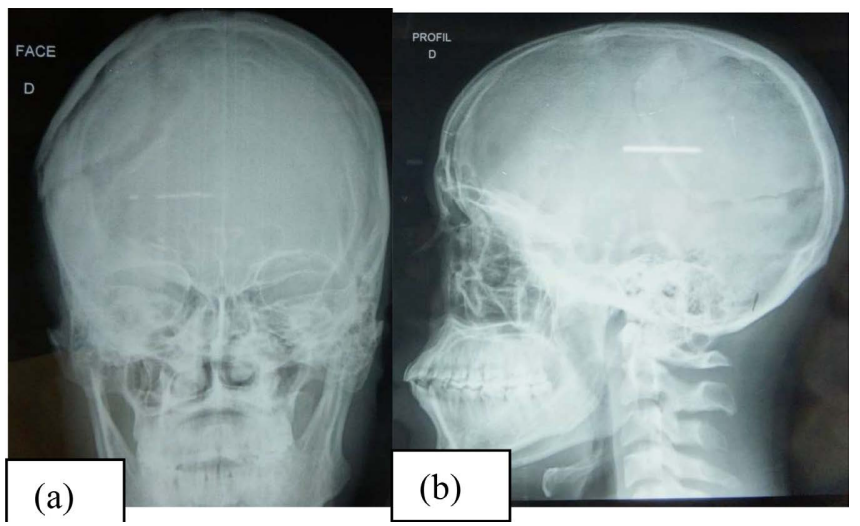

Figure 9. (a) AP and (b) lateral X-ray after cosmetic cranioplasty.

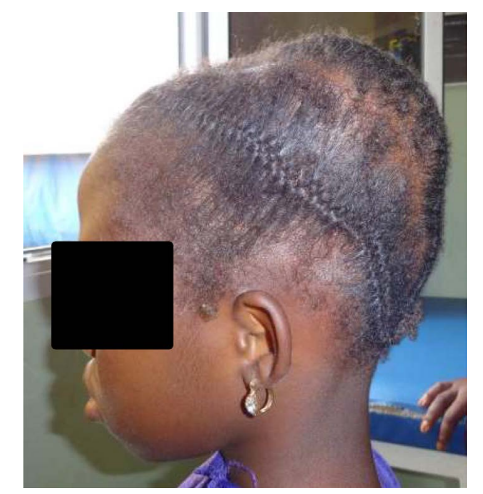

Figure 10. Clinical photograph showing the tumefaction. 


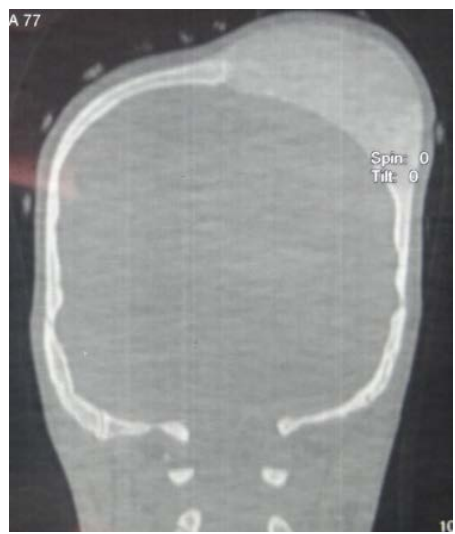

Figure 11. CT scan with FD appearance of the left parietal bone.

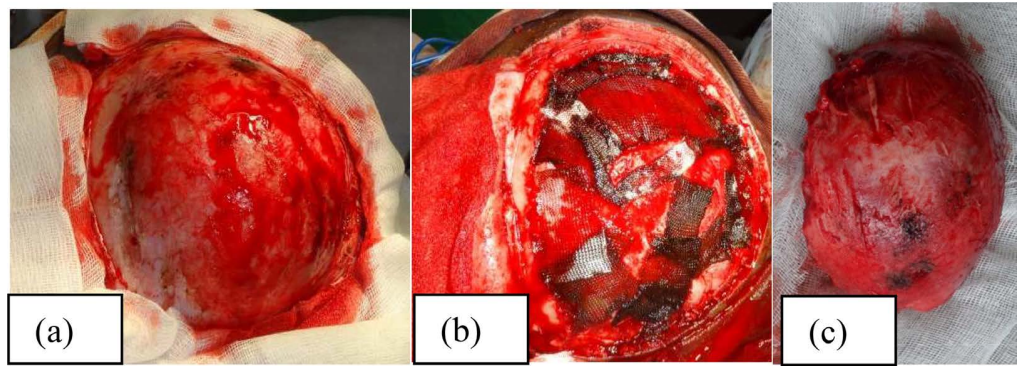

Figure 12. Operative view (a) before resection, (b) after resection, (c) bone flap.

\section{Discussion}

We described four cases of CFD who required surgical treatment. FB represents $2.5 \%$ of all bone lesions and 7\% of all benign bone tumors [5]. Both sexes are equally affected by the disease. Three of our 4 patients were male. The pathology typically occurs in the first or second decade in life. In most cases of CFD, the first clinical manifestation is a slow growing mass causing craniofacial asymmetry. That was confirmed in our four patients. In case 2, the patient was complaining with moderate headache but he had big concern with his facial asymmetry. The most clinical symptoms recorded in the literature are loss of hearing, blindness, pathological fractures, facial paralysis, proptosis, orbital dystopia, strabismus, tinnitus, epiphora and nasal obstruction [9]. These symptoms are related to the location of the disease. The diagnosis can be made with X-ray and CT-scan. Radiologically, "ground-glass" appearance with loss of the normal trabecular pattern is pathognomonic for the disease [10]. The lesions with radiologically similar appearance such as ameloblastic fibroma, osseous dysplasia, eosinophilic granuloma, chronic sclerosing osteomyelitis and central giant cell granuloma should be considered in differential diagnosis. FD is a progressive, slow rowing disease with tendency to regress after puberty. Some authors recommend surgical treatment after puberty [11]. Surgical treatment is recommended because of symptom and/or important aesthetical discomfort. In our four patients, the main indication of surgical resection was aesthetical discomfort. For that, radical resection was performed in all cases and cosmetic craniop- 
lasty with acrylic bone cement was planned after puberty. In case 3, cranioplasty performed one year later allowed to have a good aesthetic result. The other three patients are still waiting for the cranioplasty. Radical resection is the only curative technique and prevents the recurrence of the disease [12]. According to the classification suggested by Chen [13], ours patients were classified Zone 2, in which, intervention is optional. The radical excision was made in all cases because of aesthetical discomfort.

\section{Conclusion}

Fibrous Dysplasia is a benign slow growing disease that may cause as well as clinical symptom and aesthetical discomfort. Radical resection, if possible, is the only technique to obtain resolution of the disease.

\section{Conflicts of Interest}

The authors declare no conflicts of interest regarding the publication of this paper.

\section{References}

[1] Robinson, C., Collins, M.T. and Boyce, A.M. (2016) Fibrous Dysplasia/McCune-Albright Syndrome: Clinical and Translational Perspectives. Current Osteoporosis Reports, 14, 178-186. https://doi.org/10.1007/s11914-016-0317-0

[2] Lichtenstein, L. and Jaffe, H.L. (1942) Fibrous Dysplasia of Bone. Archives of Pathology, 33, 777-816.

[3] DiCaprio, M.R. and Enneking, W.F. (2005) Pathophysiology, Evaluation, and Treatment. The Journal of Bone \& Joint Surgery, 87, 1848-1864. https://doi.org/10.2106/00004623-200508000-00028

[4] Eversole, L.R., Sabes, W.R. and Rovin, S. (1972) Fibrous Dysplasia: A Nosologic Problem in the Diagnosis of Fibro Osseous Lesions of the Jaws. Journal of Oral Pathology \& Medicine, 1, 189-220. https://doi.org/10.1111/j.1600-0714.1972.tb01659.x

[5] Riddle, N.D. and Bui, M.M. (2013) Fibrous Dysplasia. Archives of Pathology \& Laboratory Medicine, 137, 134-138. https://doi.org/10.5858/arpa.2012.0013-RS

[6] Ricalde, P. and Horswell, B.B. (2001) Craniofacial Fibrous Dysplasia of the Fronto-Orbital Region: A Case Series and Literature Review. Journal of Oral and Maxillofacial Surgery, 59, 157-167. https://doi.org/10.1053/joms.2001.20487

[7] MacDonald-Jankowski, D. (2009) Fibrous Dysplasia: A Systematic Review. Dentomaxillofacial Radiology, 38, 196-215. https://doi.org/10.1259/dmfr/16645318

[8] Wang, H., Sun, X., Liu, Q., et al. (2014) Endoscopic Resection of Sinonasal Ossifying Fibroma: 31 Cases Report at an Institution. European Archives of Oto-Rhino-Laryngology, 271, 2975-2982. https://doi.org/10.1007/s00405-014-2972-z

[9] Lee, J.S., FitzGibbon, E.J., Chen, Y.R., et al. (2012) Clinical Guidelines for the Management of Craniofacial Fibrous Dysplasia. Orphanet Journal of Rare Diseases, 7, S2. https://doi.org/10.1186/1750-1172-7-S1-S2

[10] Gupta, D., Garg, P. and Mittal, A. (2017) Computed Tomography in Craniofacial Fibrous Dysplasia: A Case Series with Review of Literature and Classification Update. The Open Dentistry Journal, 30, 384-403. https://doi.org/10.2174/1874210601711010384 
[11] Fattah, A., Khechoyan, D., Phillips, J.H. and Forrest, C.R. (2013) Paediatric Craniofacial Fibrous Dysplasia: The Hospital for Sick Children Experience and Treatment Philosophy. Journal of Plastic, Reconstructive \& Aesthetic Surgery, 66, 1346-1355. https://doi.org/10.1016/j.bjps.2013.05.031

[12] Valentini, V., Cassoni, A., Marianetti, TM., et al. (2009) Craniomaxillofacial Fibrous Dysplasia: Conservative Treatment or Radical Surgery? A Retrospective Study on 68 Patients. Plastic and Reconstructive Surgery, 123, 653-660.

https://doi.org/10.1097/PRS.0b013e318196bbbe

[13] Chen, Y.R. and Noordhoff, M.S. (1990) Treatment of Craniomaxillofacial Fibrous Dysplasia: How Early and How Extensive? Plastic and Reconstructive Surgery, 86, 835-842. https://doi.org/10.1097/00006534-199011000-00002 